\title{
Preface
}

\section{Distributed Parameter Systems in Immunology}

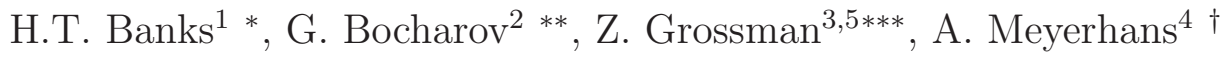 \\ ${ }^{1}$ Center for Research in Scientific Computation, \\ Center for Quantitative Sciences in Biomedicine \\ N.C. State University, Raleigh, NC 27695-8212, USA \\ 2 Institute of Numerical Mathematics, \\ Russian Academy of Sciences, Moscow 119333, Russia \\ ${ }^{3}$ Laboratories of Immunology and Cellular and Molecular Immunology \\ National Institute of Allergy and Infectious Diseases, National Institutes of Health \\ Bethesda, MD 20892, USA \\ ${ }^{4}$ Department of Experimental and Health Sciences, Universitat Pompeu Fabra \\ Barcelona 08003, Spain \\ ${ }^{5}$ Sackler Faculty of Medicine, Tel Aviv University, Israel
}

\begin{abstract}
Keywords and phrases: mathematical model, distributed parameter systems, immunophysiological systems, virus infections
\end{abstract}

Mathematics Subject Classification: 92-02, 92-06

Mathematical modelling in immunology is about forty years old. This is a great age implying a symphony of experience, creativity and energy. Ever since, there is an increasing attempt to utilize the inherent precision of mathematics to enhance the analytical tools of basic and applied research in immunology. Despite numerous publications on application of mathematical models in immunology, the major communities of the experimental scientists and mathematicians are still disconnected. The genuine integration of modeling and simulation into the immunological mainstream remains a challenge.

The immune system represents a complex and tightly regulated set of physical, biochemical and immunological processes occurring in time and space, the outcome of which is dependent on a large number of intra- and inter-cellular parameters. Coordinating a functional immune response requires tightly regulated communication between the cells of the immune system, which occurs in the form of cell-to-cell contact and the release of soluble factors that bind cognate receptors expressed by the cells. The functioning of the immune system involves feedback regulated spatially distributed proliferation, differentiation

*Corresponding author. E-mail: htbanks@ncsu.edu

** Corresponding author. E-mail: bocharov@inm.ras.ru

*** Corresponding author. E-mail: GROSSMANZ@niaid.nih.gov

${ }^{\dagger}$ Corresponding author. E-mail: andreas.meyerhans@upf .edu

(C) EDP Sciences, 2012 
and death processes of heterogeneous cell populations. Paul's introductory overview to this special issue describes key aspects of the immune system from the point of view of one of the great authorities in the field. Of particular interest here is that one of the three levels of complexity highlighted is the important but less appreciated process of "dynamic tuning", whereby the responsiveness of each individual cell to the molecular mediators of intercellular interactions is continuously updated and adjusted.

Because of the inherent complexity of the immune system a quantitative predictive understanding of its regulation requires integration of experiments aimed at gathering data at various levels of the immune system organization and the mathematical models based upon modern powerful methods of systems analysis and computational modeling technologies. A mathematical modeling approach (using mathematical tools to complement experimentation and bioscientific theories in immunology) is most useful to represent, interpret and predict the observable immune and physiological phenomena and gain further insight (which can either be substantiated or refuted) into the dynamics of immune and physiological processes.

The special issue aims to review mathematical models of differing complexity formulated with different types of equations proposed to establish a coherent basis for the computational analysis of the dynamics of distributed parameter systems in immunology using next generation mathematical models. The practical implementation of such an approach raises genuine challenges in a range of applicable mathematics (e.g., formulation of models for hierarchical and distributed systems, identification of the best mathematical description, representation of biological variation in the models). The study by Banks and $\mathrm{Hu}$ [3] compares two approaches to model growth variability in physiologically structured population models, one resulting in Fokker-Planck formulations and the other one entailing a probabilistic structure on the set of the physiological parameters across the entire population. The relationship between these two conceptually distinct approaches is examined. Proliferating cell populations are the central subject of studies in immunology. An overview of mathematical models of differing complexity (random birthdeath-, fixed cycle-, Smith-Martin-, probabilistic- and label-structured PDE models) developed so far for describing the cell growth kinetics and the estimation of the turnover parameters is reviewed in the paper by Banks and Thompson [4]. It covers a broad range of issues related to data collection (based on using intracellular dyes such as CFSE and flow cytometry), model formulation, parameter estimation and ranking of different mathematical models. The measurement of CFSE dilution by flow cytometry is a powerful experimental tool for gathering the data which are used to calibrate the mathematical models. The critical aspects of the data variability which may be important to consider when modelling dye dilution data sets are reviewed in the study by Quah et al. [5] This contribution by the founders of the use of vital fluorescent dyes in immunology represents a nice example of a fruitful feedback coming from the use of mathematical models in the analysis of heterogeneous cell populations.

Mathematical modelling of immunological phenomena requires a methodology for an integrative description of various physiologically linked systems (e.g., the lymphoid tissues, peripheral organs, blood and lymph compartments). The corresponding hybrid models are still rare. The survey by Kappel [6] addresses basic methodologies used in mathematical physiology to model the joint dynamics of cardiovascular and respiratory systems in humans. It provides an extensive discussion of parameter estimation and model validation issues. Protection against life-threatening infections is a major function of the immune system. HIV infection inspired an extensive research involving mathematical modeling. The paper by Bocharov et al. [7] reviews the fundamental biology and current concepts of HIV infection pathogenesis and the mathematical models proposed for studying HIV infection dynamics. A remarkable potential of the human immunodeficiency virus to change via mutation, multi-infection and recombination processes limits the ability of the immune system to control the infection as well as the efficiency of the antiviral treatments. The evolving virus population represents a heterogeneous collection of the genomes known as viral quasispecies. The review by Domingo and Perales [8] introduces a new perspective on the use of the mathematical theory of quasispecies dynamics in designing novel approaches to antiviral strategies (e.g. based on lethal mutagenesis). Virus replication in infected cells is usually described by simplistic exponential growth- or target cell-virus type frameworks. However, the set of intracellular biochemical processes involved in replication of viruses is enormous and deserves special attention in the next 
generation mathematical models of the within-host infection dynamics. The paper by Perez-Vilaro and coworkers [9] describes some of the most important cell components known as virus host factors which affect the virus replication. This review is focused on HIV and hepatitis C virus, representing two major human pathogens.

Overall, the contributed articles focus on overviews and 'big picture' ideas. The special issue continues previous efforts of the special issue on Mathematics applied to Immunology published in the Journal of Computational and Applied Mathematics (2005). We hope that the present one will foster the coming of the golden age of this field.

We would like to acknowledge the support from the Spanish Ministry of Education, Culture and Sports during the work on this special issue. The generous help of the referees from Germany, Israel, Spain, UK, USA and United Arab Emirates is much appreciated. Finally, we thank the Editor-in-Chief of the Mathematical Modelling of Natural Phenomena journal Dr. Vitaly Volpert for making this special issue possible.

Guest Editors: H.T Banks, G. Bocharov, Z. Grossman, A. Meyerhans

\section{References}

[1] H.T Banks, G. Bocharov, Z. Grossman, A. Meyerhans. Preface

[2] W.E. Paul. The Immune System - Complexity Exemplified

[3] H.T. Banks, S. Hu. Propagation of growth uncertainty in a physiologically structured population

[4] H.T. Banks, W.C. Thompson. Mathematical models of dividing cell populations: application to CFSE data

[5] Ben J.C. Quah, A. Bruce Lyons, C.R. Parish. The use of CFSE-like dyes for measuring lymphocyte proliferation: experimental considerations and biological variables

[6] F. Kappel. Modeling the dynamics of the cardiovascular-respiratory system (CVRS) in humans, a survey

[7] G. Bocharov, V.A. Chereshnev, I. Gainova, S. Bazhan, B. Bachmetyev, J.M. Argilaguet, J.P. Martinez, A. Meyerhans. Human Immunodeficiency Virus infection: from biological observations to mechanistic mathematical modelling

[8] E. Domingo, C. Perales. From quasispecies theory to viral quasispecies: how complexity has permeated virology

[9] G. Pérez-Vilaró, J. Jungfleisch, V. Saludes, N. Scheller, M. Giménez-Barcons, J. Díez. Host factors in viral life cycles 